\title{
C5/C6 - les bons mots pour en parler
}

\section{Rouven Porz}

PD Dr phil., biol. dipl., responsable du Service ethique chez Insel Gruppe AG (Berne), chercheur invité de la VU Amsterdam, Président de la European Association of Centres of Medical Ethics (EACME) et membre de la rédaction de la rubrique Ethique du BMS

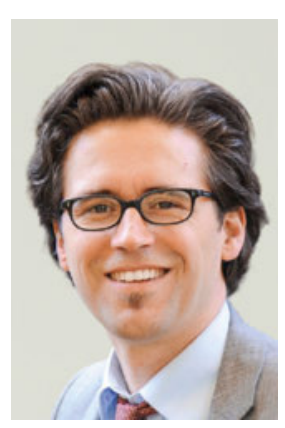

Je souffre d'une hernie discale. Consultation médicale, imagerie, organisation du traitement, jusque-là, tout va bien. Je fais confiance à mon médecin. C'est alors qu'un voisin rencontré devant chez moi me pose des questions en me voyant marcher voûté et le pas hésitant. "Oui, j’ai une hernie discale», lui ai-je répondu. "Ah bon, où exactement»? "Euh, vers le cou, ou les épaules, dans cette zone.» Il m’a regardé, consterné. Il a insisté encore deux ou trois fois: il voulait savoir exactement et m'assaillait de chiffres et de lettres. J'ai bafouillé une excuse et ai filé dans l'entrée. J’ai trouvé bizarre cet intérêt soudain pour la médecine alors qu'il était menuisier. Je suis rentré chez moi et ai demandé à ma femme: «Dis-moi, où se situe ma hernie discale exactement?» «C5/C6» m'a-t-elle répondu laconiquement. Ah, c'est ce que mon voisin aurait voulu entendre.

Le lendemain, je rencontre un collègue de travail. Il me demande comment ça va et une fois encore je réponds spontanément: «’’ai une hernie discale.» Zut, me dis-je en même temps, je ne voulais plus en parler. "Où exactement?», me demande-t-il aussitôt, dégainant plus vite que son ombre. Je lui réponds d'un air dédaigneux: «C5/C6». Moi qui n'étais pas médecin, je trouvais déjà que l'échange prenait une tournure risquée. «Et tu as commencé le sport?» «Hein? Comment?» Je ne comprenais pas bien la question et tentai une sortie ironique: «Oui, je viens justement de m'inscrire à un cours de kitesurf dans l'Aar.» J'ai vraiment dû réprimer un fou rire, mais mon humour est mort dans l'œuf, car mon interlocuteur ne trouvait là-dedans rien de drôle ni d'ironique. Il ne me laissa pas partir avant de m'avoir expliqué pendant cinq minutes les bénéfices du sport et de la physiothérapie quand on avait des problèmes de hernie discale. La physiothérapie je le savais, j'aurais pu le dire, mais le sport franchement.

Le soir, à la physiothérapie, je rencontre une compagne d'infortune. Même diagnostic, hernie discale. Nous avons bien papoté dans la salle d'attente. Je me suis entendu dire: "Oui, moi c'est la C5/C6». Effectivement, maintenant je dois suivre une physiothérapie. C'est un fait, il faut désormais envisager de faire du sport. Lentement, j'en suis venu à parler de ma maladie. Puis j'ai raconté qu'un charmant médecin m'avait planté une immense seringue dans le cou, juste à l'endroit de la hernie. La seringue m'avait fait peur. Mais le médecin m’a rassuré, ce que j'ai apprécié. "Comment ça une seringue? On vous a fait une infiltration de cortisone?» J'ai acquiescé, hésitant et incertain. Est-ce qu'on est un patient uniquement à partir du moment où on peut expliquer sa maladie avec des termes techniques incompréhensibles? Je ne pouvais plus m'enfuir de la salle d'attente. La conversation a continué. «Et maintenant, Monsieur Porz, vous poursuivez uniquement avec un traitement conservateur ou vous prévoyez une intervention chirurgicale?» Pardon? Un traitement conservateur? Une intervention chirurgicale?

Le soir, j'ai dû à nouveau me tourner vers mon épouse. Le lecteur remarquera qu'elle s'y connaît. «Tu commences par un traitement conservateur, et si ça ne marche pas, nous envisagerons l'opération.» D'accord, ma femme étant médecin, elle peut s'exprimer comme ça. Mais pourquoi les profanes parlent-ils tous en termes si savants? Le sujet mérite réflexion. Sont-ils tous obligés d'utiliser ce jargon? A moins que nos médecins ne fassent pas assez d'efforts pour abandonner leur langage technique et expliquer aux patients avec des mots simples ce qui ne va pas. Soudain, cela sonne à la porte. Un autre voisin me demande si je peux l'aider rapidement à décharger son nouveau meuble car il ne peut pas le sortir tout seul de sa voiture. Je lui explique: "C'est trop bête, j'ai malheureusement une hernie discale». Il me regarde avec de grands yeux. Et là, je m'entends lui répondre: «C5/C6, j’ai eu une infiltration, j'essaie maintenant un traitement conservateur avec de la physio.» Il semble satisfait. Il me souhaite bonne chance. Pas de leçon. Pas de demande de détails. Pas de trouble. Tout est resté calme. C'est bien, pensai-je. Je me dis que c'est une bonne chose que je connaisse ma propre histoire. Je ne peux pas m'empêcher de sourire. Une bonne maladie mérite manifestement qu'on en parle dans des termes médicaux. 